\title{
Epidemia de febre amarela na bacia do Rio Doce: análise de fatores ambientais, epidemiológicos e efeitos indiretos do rompimento da barragem de Fundão (Samarco S/A)
}

\author{
Gabriel de Oliveira Contini Pereira ${ }^{1}$ \\ Elvira Carolina Bicalho ${ }^{2}$ \\ Jaquelline Carla Valamiel de Oliveira e Silva ${ }^{3}$ \\ Sofia Luiza Brito ${ }^{4}$
}

\begin{abstract}
RESUMO
O rompimento da barragem de rejeitos de Fundão (Samarco S/A no município de Mariana - MG), em novembro de 2015 , liberou 55 milhões $\mathrm{m}^{3}$ de rejeitos, provocando a morte da biota aquática na calha central do Rio Doce e danos nos ecossistemas adjacentes. No início de 2017, iniciou-se uma epidemia de febre amarela em Minas Gerais, com grande parte das ocorrências na região da bacia do Rio Doce. Assim, este trabalho buscou verificar a existência de relação entre os dois fatos ocorridos. Os dados epidemiológicos e de monitoramento da água foram relacionados por meio de Análise de Componentes Principais (ACP), não sendo identificada relação direta entre o dano ambiental e a epidemia. Todavia, fatores como a dizimação dos predadores do mosquito vetor, a proximidade entre o homem e as florestas, e a baixa cobertura vacinal da população foram também considerados como aspectos relevantes. Desse modo, esse estudo analisou os fatores que devem ser considerados em conjunto, no intuito de compreender o aumento da febre amarela na Bacia do Rio Doce após o rompimento da barragem de Fundão.
\end{abstract}

Palavras-chave: Lama. Qualidade da água. Haemagogus spp. Epizootia.

\footnotetext{
${ }^{1}$ Graduado em Engenharia Ambiental pela Universidade do Estado de Minas Gerais - UEMG. Pós-Graduado em Docência com ênfase em Educação Básica pelo Instituto Federal Minas Gerais - IFMG. Atua como professor de Educação Básica, MG, Brasil. E-mail: continigabriel@gmail.com.

${ }^{2}$ Graduada em Engenharia Ambiental pela Universidade do Estado de Minas Gerais - UEMG. Pós-Graduada em Gestão Pública e de Pessoas pela Faculdade Única de Ipatinga - FUNIP, MG, Brasil. E-mail: elviracbicalho@gmail.com.

${ }^{3}$ Graduada em Ciências Biológicas pela Universidade Federal de Ouro Preto - UFOP. Mestra e Doutora em Imunoparasitologia pela UFOP. É professora da Universidade do Estado de Minas Gerais - UEMG, Unidade João Monlevade, MG, Brasil. E-mail: jackvalamiel@yahoo.com.br.

${ }^{4}$ Graduada em Ciências Biológicas pela Universidade Federal de Minas Gerais - UFMG. Mestra e Doutora em Ecologia pela UFMG. É professora da Universidade do Estado de Minas Gerais - UEMG, Unidade Ubá, MG, Brasil.E-mail: sofialuizabrito@gmail.com.
} 


\title{
Yellow fever epidemic in Rio Doce basin: analysis of environmental and epidemiological factors and indirect effects of Fundão dam break (Samarco S/A)
}

\begin{abstract}
The rupture of the Fundão tailings dam (Samarco S/A in the county of Mariana -MG), which took place on November, 2015, released 55 million $\mathrm{m}^{3}$ of mud, causing the death of the aquatic biota in the central channel of Rio Doce and damage to adjacent ecosystems. At the beginning of 2017, a yellow fever epidemic started in Minas Gerais, mainly in rural areas, with most of the occurrences in the region of the Rio Doce basin. Thus, the present work aimed to verify the existence of a relationship between these two facts that occurred. Data on public health and water monitoring were related using Principal Component Analysis (PCA), but there was no direct relationship between environmental damage and the yellow fever epidemic. However, factors such as decimation of predators of disease vector, proximity of humans to forests, decrease in larvaphagous fish and low vaccination coverage of population were also considered as relevant aspects. Thus, this study analysed the factors that should be considered together, in order to understand the increase in yellow fever in Rio Doce basin after Fundão dam break.
\end{abstract}

Keywords: Mud. Water quality. Haemagogus spp. Epizootic.

Artigo recebido em: 08/06/2020

Aceito em: 05/11/2020 


\section{INTRODUÇÃO}

A febre amarela é uma doença infecciosa, imunoprevenível, causada por vírus e transmitida pela picada de vetores infectados. Apresenta dois ciclos de transmissão: urbano e silvestre, com evolução clínica semelhante em ambas. No ciclo urbano, o vírus é transmitido através do mosquito Aedes aegypti (L.) (Diptera: Culicidae), o mesmo transmissor da dengue, e utiliza o homem como hospedeiro. Já no ciclo silvestre, em áreas florestais, os vetores da doença são principalmente os mosquitos Haemagogus spp e Sabethes spp (Diptera: Culicidae), que são capazes de transmitir o vírus para os macacos, hospedeiro natural do vírus nesse ambiente (FUNDAÇÃO OSWALDO CRUZ, 2017b, 2017c).

Os gêneros supracitados apresentam ampla distribuição geográfica, todavia os Sabethes spp são mais seletivos em sua distribuição. Estes vetores se concentram nos locais de vegetação preservada, enquanto os Haemagogus spp podem ser avistados ainda nos arredores das florestas, onde os Sabethes ssp. geralmente não se aventuram (FUNDAÇÃO OSWALDO CRUZ, 2017a). Possuem hábitos estritamente silvestres, só picando pessoas dentro de seu nicho ecológico, de modo que a distribuição da doença é limitada pela distribuição desses vetores. Seu padrão de transmissão depende da dinâmica de multiplicação do vírus, da ecologia e do comportamento de seus vetores e de seus hospedeiros, incluindo a imunidade (COSTA \& CALADO, 2016).

As fêmeas são primatófilas, ou seja, se alimentam preferencialmente em macacos e, secundariamente, no homem. Apresentam atividade diurna, período em que ocorre a transmissão da febre amarela em pessoas que adentram matas. Apresentam hábitos como acrodendrofilia (tendência de certos insetos silvestres a viver ao nível do topo ou copa das árvores), com preferência pela localização de criadouros em aberturas nos troncos das árvores (NEVES et al., 2005; VASCONCELOS, 2003). Algumas características ambientais são muito importantes para a manutenção e consequente reprodução do mosquito, como o tipo do clima. O clima tropical (com temperaturas elevadas e chuvas normalmente abundantes no verão), presente na maior parte do Brasil, favorece a reprodução de mosquitos, sendo assim constantes os casos diagnosticados de doenças transmitidas por esses vetores no país (REY, 2001).

Segundo informações do Ministério da Saúde (2017), a febre amarela urbana deixou de ser registrada no Brasil desde 1942. A partir desta data, só houve casos da doença na 
forma silvestre, sendo que, até o ano de 1999, os focos endêmicos se localizavam nos estados da região Norte, Centro-Oeste, pequenas áreas do Maranhão e região oeste de Minas Gerais. Em 2000, casos da doença em humanos ocorreram fora de áreas endêmicas clássicas, que possuíam cobertura florestal intacta. Investigações epidemiológicas demonstraram que esses casos ocorreram em locais com alto grau de desmatamento e também em áreas rurais onde a vegetação nativa foi transformada em remanescentes escassos, como mata ciliar ou áreas mais distantes dos rios, cercadas por pastagens.

Desde então, observa-se que as epizootias (ocorrência de doença em população animal não humana) ocorrem em remanescentes florestais que sofrem constantemente a invasão de animais domésticos e também de humanos (CAMARGO-NEVES et al., 2005). Com base nessa observação, o Ministério da Saúde, ao analisar a doença em uma determinada região, passou a considerar também fatores externos como a proximidade desta região a ecossistemas, o trânsito de pessoas e de animais domésticos para a região silvestre, a circulação viral na região, dentre outros (MINISTÉRIO DA SAÚDE, 2017).

Segundo a Secretaria de Estado de Saúde de Minas Gerais (2017), as últimas ocorrências de casos humanos de febre amarela no estado haviam sido registradas em 2008 e 2009, desde então, as notificações destes casos permaneceram silenciosas. Porém, no início do ano de 2017, o estado de Minas Gerais passou a registrar sucessivos casos da doença, sobretudo nas áreas rurais. Conforme informações do boletim sobre a investigação de casos suspeitos da doença, divulgado pela Secretaria de Estado de Saúde (SES, 2017), cerca de metade dos casos ocorreram em municípios localizados na bacia do Rio Doce.

Esta região foi uma das mais afetadas pelo rompimento da barragem de rejeitos da empresa Samarco S/A ocorrido no município de Mariana, em Minas Gerais, em 5 de novembro de 2015. O acidente foi então considerado como o pior desastre ambiental brasileiro, uma vez que houve liberação de mais de 55 milhões de $\mathrm{m}^{3}$ de resíduos ao longo de $663 \mathrm{~km}$ nos rios Gualaxo do Norte, Carmo e Doce. Este episódio gerou severos danos socioambientais por todas as cidades presentes no percurso longitudinal até chegar no litoral capixaba, onde adentrou $80 \mathrm{~km}^{2}$ no mar (SEDRU, 2016).

A Bacia do Rio Doce possui $98 \%$ de sua área inserida no bioma da Mata Atlântica, um dos 34 hotspots mundiais para a conservação da biodiversidade devido aos altos níveis de espécies endêmicas e alteração antrópica (MYERS et al., 2000). Entretanto, o fluxo de rejeitos proveniente do acidente apresentou-se de forma expressiva, prejudicando ainda mais essa região. O desastre foi classificado pela Defesa Civil de Minas Gerais como nível IV, 
isto é, "desastre de porte muito grande". Quanto aos danos ambientais, a liberação da lama provocou a pavimentação de uma grande área, infertilidade do solo, morte da vegetação, danos aos recursos hídricos com consequente morte de peixes e desequilíbrio da cadeia alimentar, assoreamento, desvio dos cursos d'água e soterramento de nascentes (SANTOS, 2017).

Neste contexto, considerando o aumento da febre amarela, no início de 2017, em municípios localizados na bacia do Rio Doce, este trabalho teve como objetivo avaliar a possível correlação desses casos com o rompimento da barragem de Fundão ocorrido em novembro de 2015.

\section{METODOLOGIA}

Grande parte dos dados pertinentes a este estudo são de ampla divulgação nos canais de comunicação relacionados a fatores ambientais, biológicos e de saúde pública, sendo utilizados bancos de dados públicos como INFOHIDRO, IGAM, INMET, IBGE, Ministério da Saúde, SES-MG, além de material publicado sobre as características da doença estudada, a fim de se estabelecer relação entre o surto de febre amarela e o desastre ambiental provocado pelo rompimento da barragem de Fundão da Samarco S/A.

Os dados epidemiológicos (número de casos, óbitos e epizootias) foram obtidos por meio dos informes da Secretaria do Estado de Saúde, SES-MG, referentes ao $4^{\circ}$ trimestre de 2014, aos anos de 2015 e 2016 e ao primeiro trimestre de 2017. Estes dados são utilizados para a associação das estações de monitoramento da qualidade de água às cidades que apresentaram casos de febre amarela.

O monitoramento da qualidade das águas na bacia hidrográfica do Rio Doce contempla atualmente 64 estações de amostragem, onde são realizadas coletas e análises laboratoriais com periodicidade trimestral e avaliação de aproximadamente 50 parâmetros físicoquímicos e hidrobiológicos, dentre eles o Índice de Qualidade da Água (IQA), Contaminação por Tóxicos (CT) e Índice de Estado Trófico (IET), além da presença de certas substâncias. Estes dados são divulgados, trimestralmente, pelo portal InfoHidro (Informações sobre Recursos Hídricos) (IGAM, 2017).

Neste estudo, foram obtidos dados dos relatórios do monitoramento da qualidade das águas superficiais no Estado de Minas Gerais no mesmo intervalo de tempo das amostras epidemiológicas (IGAM, 2015a, 2015b, 2016a, 2016b, 2016c, 2016d, 2017), utilizando 
dados de 13 estações de amostragem do monitoramento do Rio Doce localizadas desde o seu afluente Rio do Carmo até a cidade de Aimorés, antes do exutório para o estado do Espírito Santo.

Para possibilitar a relação da quantidade de casos notificados e confirmados (SESMG) com a qualidade da água de cada região foi necessário verificar a localização das estações de amostragem de monitoramento do IGAM com o intuito de associá-las, de acordo com a proximidade, às cidades com epidemia de febre amarela (Tabela 1).

Tabela 1: Relação entre estações de monitoramento do IGAM e cidades com casos de febre amarela em 2017 na bacia do Rio Doce

\begin{tabular}{|c|c|c|c|}
\hline $\begin{array}{l}\text { Código } \\
\text { IGAM }\end{array}$ & Cidades associadas & $\begin{array}{l}\text { Código } \\
\text { IGAM }\end{array}$ & Cidades associadas \\
\hline RD071 & Alvinópolis & \multirow{3}{*}{ RD033 } & Mesquita \\
\hline RD072 & Ponte Nova & & Belo Oriente \\
\hline RD019 & $\begin{array}{c}\text { Raul Soares } \\
\text { Nova Era }\end{array}$ & & $\begin{array}{c}\text { Naque } \\
\text { Dom Cavati }\end{array}$ \\
\hline \multirow{5}{*}{ RD023 } & Timóteo & RD083 & Tarumirim \\
\hline & Vargem Alegre & RD044 & Alpercata \\
\hline & Entre Folhas & RD045 & Governador Valadares \\
\hline & Ubaporanga & RD053 & Itanhomi \\
\hline & Caratinga & RD058 & Conselheiro Pena \\
\hline \multirow{3}{*}{ RD035 } & Ipatinga & & Resplendor \\
\hline & $\begin{array}{l}\text { Santana do Paraíso } \\
\text { Coronel Fabriciano }\end{array}$ & RD059 & $\begin{array}{c}\text { Santa Rita do Itueto } \\
\text { Itueta }\end{array}$ \\
\hline & Ipaba & RD067 & Aimorés \\
\hline
\end{tabular}

Fonte: IGAM (2015a).

Para verificar as diferenças entre IQA, IET e CT entre os diferentes trimestres avaliados neste trabalho foi realizada uma Análise de Variância (ANOVA), seguida do teste de Tukey. Já o efeito do rompimento da barragem de Fundão na bacia do Rio Doce sobre a epidemia de febre amarela, em 2017, foi verificado por uma Análise de Componentes Principais (ACP) entre os índices de qualidade de água monitorados pelo IGAM e os números de casos de febre amarela nos municípios da região (SES-MG). Após análise prévia dos dados, alguns parâmetros foram retirados da análise, como número de óbitos, devido à descontinuidade das informações. As análises de Variância e de Componentes Principais foram realizadas no software Statistica da Statsoft, versão teste.

Buscando outros possíveis fatores para a ocorrência da epidemia de febre amarela e considerando que a doença é prevenida através da imunização, foram analisados os dados de cobertura vacinal, obtidos junto ao Sistema de Informações do Programa Nacional de 
Imunizações (SIPNI), sistema que faz parte do Departamento de Informática do SUS (DATASUS).

\section{RESULTADOS E DISCUSSÃO}

\subsection{Dados epidemiológicos para febre amarela}

No período compreendido entre janeiro e agosto de 2017, o número de casos notificados de febre amarela apresentou um aumento de 35 vezes no estado, caracterizando um surto epidêmico, o que pode ser verificado na Figura 1.

Devido ao fato da febre amarela não possuir registros históricos de grandes índices de infecção na região mineira, a rápida evolução da doença observada durante o ano de 2017 gerou grande preocupação na população e nas autoridades de saúde. No ano anterior, por

exemplo, foram registrados apenas 40 casos ao longo dos 12 meses. É possível perceber que houve um aumento abrupto no número de casos notificados ao longo do mês de janeiro até o mês de fevereiro, quando os números começam a estabilizar. Nota-se ainda, entre os meses de junho e agosto, um grande aumento nas notificações, que, segundo a Secretaria de Estado de Saúde (2017), é reflexo do intenso trabalho de consolidação dos dados oriundos de diferentes fontes de informação e também de uma maior sensibilidade por parte da vigilância em saúde. Desse modo, o aumento das notificações, não significa aumento real no número de casos.

Em relação aos casos confirmados, percebe-se também que aumentaram ao longo dos meses avaliados, entretanto, este aumento foi menor e menos acentuado que os casos notificados no mesmo período. Já em relação aos números de óbitos confirmados, foi observado um aumento gradativo ao longo dos períodos, sendo que, nos últimos meses, os óbitos tiveram uma tendência a se estabilizar. 
Figura 1: Progressão do surto de febre amarela em Minas Gerais no ano de 2017

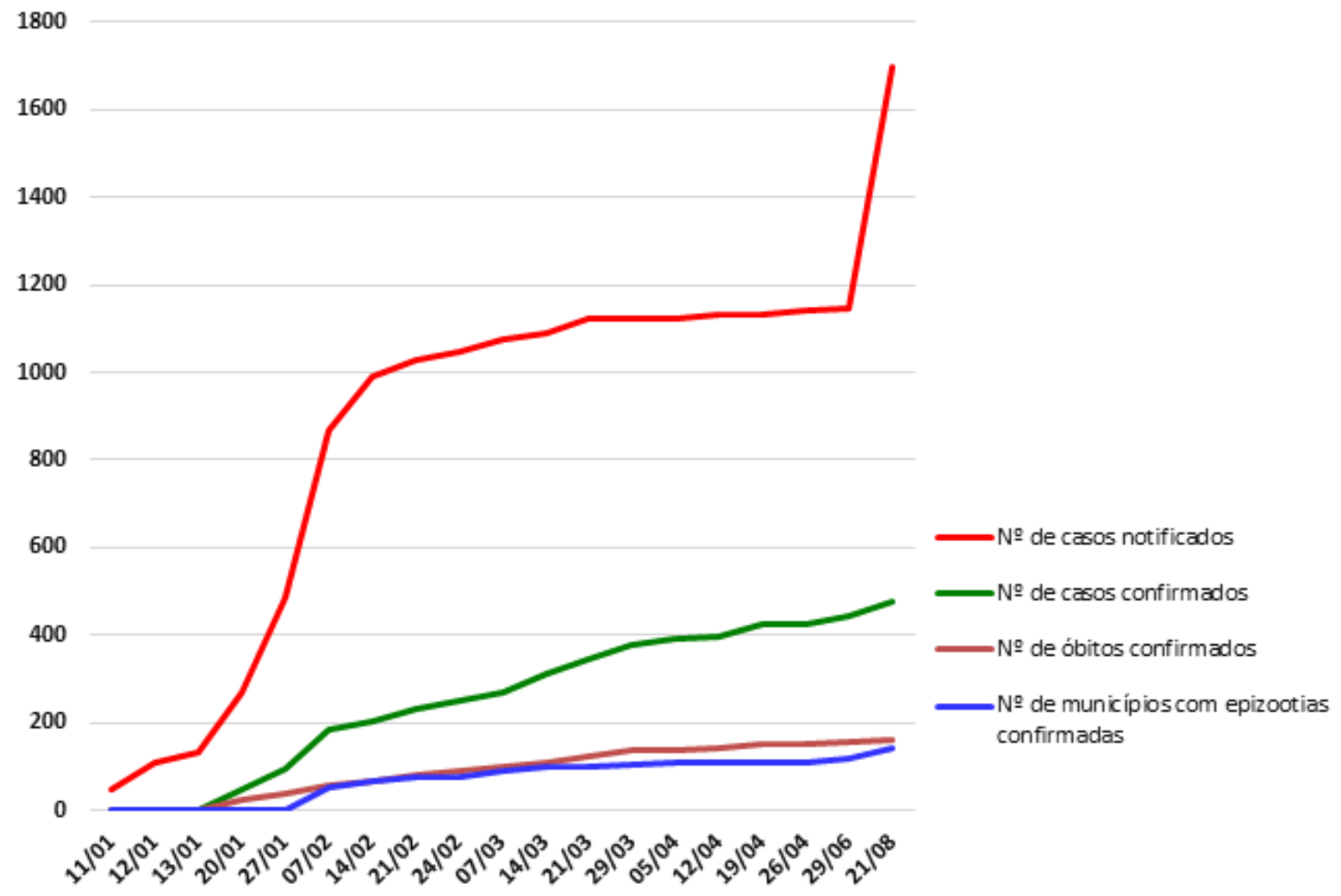

Fonte: MINISTÉRIO DA SAÚDE (2017).

A Figura 2 demonstra a distribuição da epidemia de febre amarela por região de saúde, em que é possível verificar que a maior quantidade de casos e óbitos por febre amarela ocorreu nas regiões de saúde de Teófilo Otoni, Coronel Fabriciano, Governador Valadares e Manhumirim. No presente estudo, esses dados contribuíram para a definição dos pontos de amostragem que se enquadram nos critérios de proximidade com a região da Bacia do Rio Doce, motivo pelo qual os dados da regional de saúde de Manhumirim não foram considerados na análise. 
Figura 2: Distribuição de febre amarela por Regional de Saúde em 2017

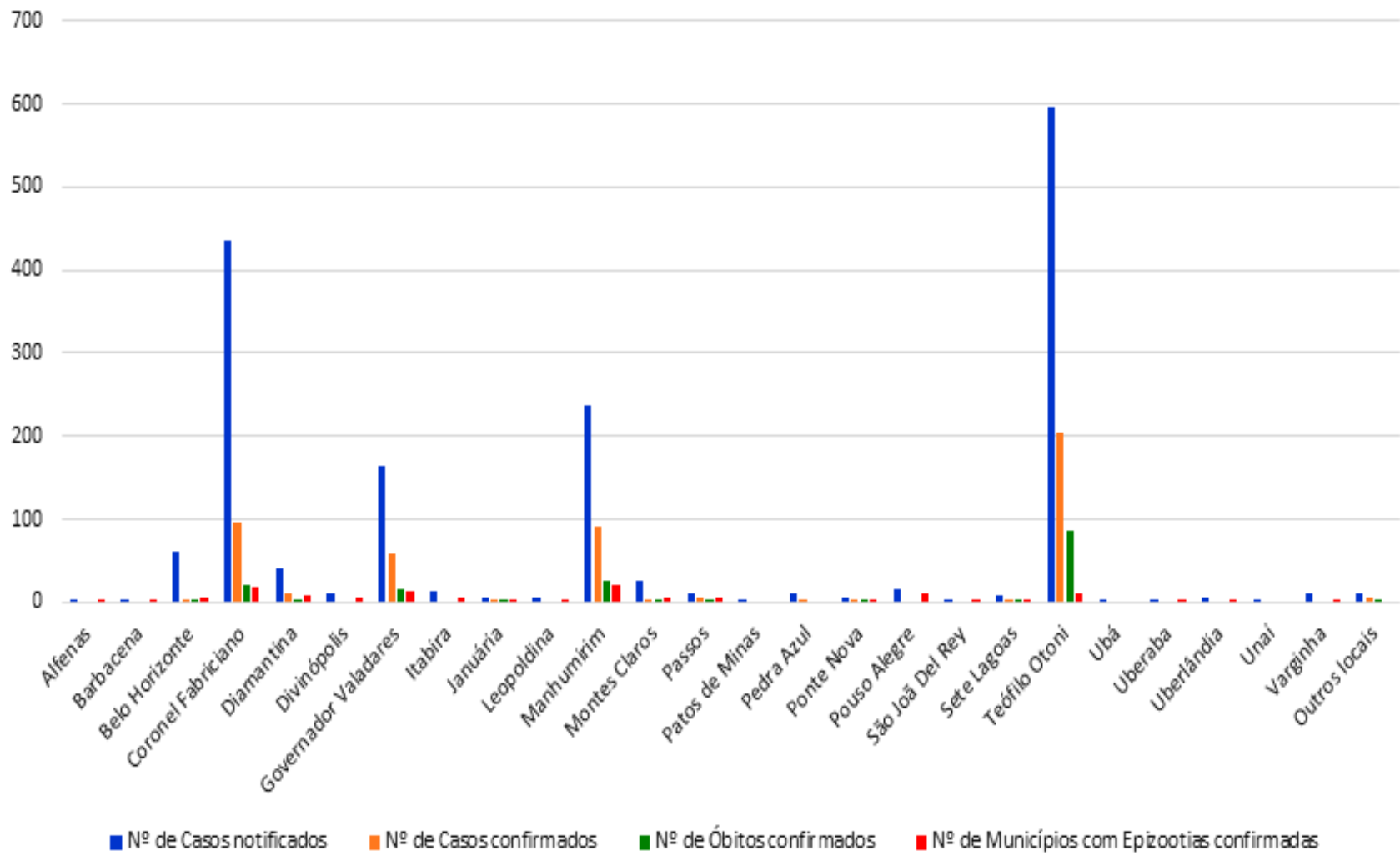

Fonte: MINISTÉRIO DA SAÚDE (2017).

\section{2 Índices de qualidade da água na Bacia do Rio Doce}

A Tabela 2 demonstra os resultados obtidos pelas análises das amostras de água para as 13 estações em três diferentes momentos: $3^{\circ}$ trimestre de 2015 (antes do rompimento da barragem), $4^{\circ}$ trimestre de 2015 (período em que ocorreu o desastre) e o $1^{\circ}$ trimestre de 2016 (após o rompimento da barragem).

Ao longo do período avaliado, ocorreu uma queda de 32,5\% do IQA (Tabela 2). Este declínio foi mais acentuado no $1^{\circ}$ trimestre de 2016, sendo uma possível consequência do rompimento da Barragem de Fundão no final do $4^{\circ}$ trimestre de 2015. Para Minas Gerais, a faixa do IQA considerada como ótima é aquela que apresenta valor igual ou superior a 90 (IGAM, 2017). Entretanto, dados publicados pelo IGAM demonstram que antes do acidente a qualidade da água do Rio Doce era considerada como boa (média de 70,58) e logo após o ocorrido ela passou a ser enquadrada como ruim $(45,74)$. 
Tabela 2: Monitoramento da qualidade das águas nas estações de coleta do Rio Doce no intervalo de $07 / 2015$ a $03 / 2016$

\begin{tabular}{|c|c|c|c|c|c|c|c|c|c|}
\hline \multirow[b]{2}{*}{ Código IGAM } & \multicolumn{3}{|c|}{ Resultado $3^{\circ}$ tri/2015 } & \multicolumn{3}{|c|}{ Resultado $4^{\circ}$ tri/2015 } & \multicolumn{3}{|c|}{ Resultado $1^{\circ}$ tri/2016 } \\
\hline & IQA & $\mathrm{CT}$ & IET & IQA & $\mathrm{CT}$ & IET & IQA & CT & IET \\
\hline RD071 - Barra Longa & 69,1 & baixa & 56,4 & 67,3 & baixa & 52,8 & 48,1 & baixa & 29,5 \\
\hline RD072 - Rio Doce & 68,2 & baixa & 48,6 & 56,4 & baixa & 54 & 43,6 & alta & 58,2 \\
\hline RD019 - Rio Casca & 78,4 & baixa & 50,6 & 61,8 & baixa & 56,4 & 45 & baixa & 32 \\
\hline RD023 - Marliéria & 79,8 & baixa & 39 & 65 & baixa & 56 & 49 & alta & 45,9 \\
\hline RD035 - Santana do Paraiso & 60,6 & baixa & 42,1 & 57,4 & média & 40 & 45 & baixa & 39 \\
\hline RD033 - Belo Oriente & 59,4 & baixa & 42,6 & 51,6 & baixa & 55,6 & 42,1 & média & 58,5 \\
\hline RD083 - Periquito & 77,7 & baixa & 49,7 & 62,5 & baixa & 60,5 & 49,3 & alta & 40,8 \\
\hline RD044 Governador Valadares I & 69,8 & baixa & 42,7 & 53,4 & baixa & 56,8 & 43,1 & alta & 44,4 \\
\hline RD045 - Governador Valadares II & 65,6 & baixa & 49,3 & 56 & baixa & 52,2 & 45,8 & alta & 58 \\
\hline RD053 - Tumiritinga & 77,5 & baixa & 51,6 & 78,7 & baixa & 50,6 & 45 & baixa & 39 \\
\hline RD058 - Conselheiro Pena & 66,9 & baixa & 51,6 & 52 & baixa & 41,2 & 45,5 & alta & 32,3 \\
\hline RD059 - Resplendor & 72,3 & baixa & 52,8 & 63 & baixa & 41,8 & 44,8 & alta & 46,8 \\
\hline RD067 - Aimorés & 72,3 & baixa & 49,6 & 54,9 & média & 43,3 & 48,3 & alta & 45,5 \\
\hline Média & 70,58 & baixa & 48,20 & 60,00 & baixa & 50,86 & 45,74 & média & 43,84 \\
\hline
\end{tabular}

IQA: Índice de Qualidade da Água

CT: Contaminação por Tóxicos IET: Índice de Estado Trófico

Fonte: IGAM (2015a, 2015b, 2016a).

Não ocorreram alterações significativas para o IET quando comparadas as médias em cada período avaliado. Foi observado um aumento de 5,52\% no trimestre em que ocorreu o derramamento de lama no Rio Doce ( $4^{\circ}$ trimestre de 2015), e assim o IET da água mantevese na classe oligotrófico $(47<$ IET $\leq 52)$, mesma classificação segundo a faixa de variação apresentada por Lamparelli (2004). Comparando as médias do IET no $1^{\circ}$ trimestre de 2016 com o último trimestre de 2015, foi observada uma queda de 13,81. Desta forma a água se enquadrou, nesse período, em uma classificação de ultraoligotrófico (IET $\leq 47$ ), com condição melhor do que se encontrava anteriormente.

Antes do rompimento da barragem, a média das 13 estações de monitoramento apresentou a CT baixa. Esse índice corresponde à ocorrência de substâncias tóxicas em concentrações que excedem em até $20 \%$ o limite de classe de enquadramento do trecho do corpo de água onde se localiza a estação de amostragem. Porém, com a presença da lama e o material tóxico nela contida, $61,54 \%$ das estações tiveram a contaminação classificada como alta no $1^{\circ}$ semestre de 2016 , já que as concentrações excederam em mais de $100 \%$ os limites permitidos.

A Análise de Variância apresentou diferenças significativas nos valores do IQA ( $\mathrm{F}=$ 24,88; $\mathrm{p}<0,0001)$ e do CT $(\mathrm{F}=20,12 ; \mathrm{p}<0,0001)$. O teste de Tukey apontou que estas 
diferenças estão nas coletas do $1^{\circ}$ e $2^{\circ}$ trimestres de 2016 com menores valores de IQA e alta Contaminação por Tóxicos.

Os dados do monitoramento do IGAM nos três trimestres antes do surto epidemiológico de febre amarela na Bacia do Rio Doce em Minas Gerais (cerca de um ano após o desastre) foram apresentados na Tabela 3.

Tabela 3: Monitoramento da qualidade das águas nas estações de coleta do Rio Doce no intervalo de $04 / 2016$ a $12 / 2016$

\begin{tabular}{|c|c|c|c|c|c|c|c|c|c|}
\hline \multirow[b]{2}{*}{ Código IGAM } & \multicolumn{3}{|c|}{ Resultado $2^{\circ}$ tri/2016 } & \multicolumn{3}{|c|}{ Resultado $3^{\circ}$ tri/2016 } & \multicolumn{3}{|c|}{ Resultado $4^{\circ}$ tri/2016 } \\
\hline & IQA & CT & IET & IQA & $\mathrm{CT}$ & IET & IQA & $\mathrm{CT}$ & IET \\
\hline RD071 - Barra Longa & 48,1 & baixa & 29,5 & 58,4 & baixa & 53,1 & 51,3 & alta & 52,8 \\
\hline RD072 - Rio Doce & 43,6 & alta & 58,2 & 61,5 & baixa & 56,3 & 53,2 & baixa & 56,7 \\
\hline RD019 - Rio Casca & 45 & baixa & 32 & 56,2 & baixa & 53,5 & 50,3 & baixa & 57,2 \\
\hline RD023 - Marliéria & 49 & alta & 45,9 & 59,7 & baixa & 51,3 & 53,9 & baixa & 48,4 \\
\hline RD035 - Santana do Paraiso & 45 & baixa & 39 & 46,8 & baixa & 48 & 45,3 & baixa & 47,1 \\
\hline RD033 - Belo Oriente & 42,1 & média & 58,5 & 46,6 & baixa & 51,8 & 47,6 & baixa & 53,7 \\
\hline RD083 - Periquito & 49,3 & alta & 40,8 & 62,7 & baixa & 55,2 & 63,2 & baixa & 56,4 \\
\hline RD044 Governador Valadares I & 43,1 & alta & 44,4 & 57,3 & baixa & 53,5 & 55,8 & baixa & 51,4 \\
\hline RD045 - Governador Valadares II & 45,8 & alta & 58 & 63,4 & baixa & 50,7 & 59,5 & baixa & 52,1 \\
\hline RD053 - Tumiritinga & 46,3 & alta & 40 & 75,3 & baixa & 54 & 67,4 & baixa & 52,7 \\
\hline RD058 - Conselheiro Pena & 45,5 & alta & 32,3 & 71,8 & baixa & 50,7 & 57,8 & baixa & 46,1 \\
\hline RD059 - Resplendor & 44,8 & alta & 46,8 & 75,5 & baixa & 53,9 & 66,6 & baixa & 57,9 \\
\hline RD067 - Aimorés & 48,3 & alta & 45,8 & 66,1 & baixa & 51,1 & 63,8 & baixa & 55,6 \\
\hline Média & 45,84 & média & 43,94 & 61,64 & baixa & 52,55 & 56,59 & baixa & 52,93 \\
\hline
\end{tabular}

IQA: Índice de Qualidade da Água

CT: Contaminação por Tóxicos

IET: Índice de Estado Trófico

Fonte: IGAM (2016b, 2016c, 2016d).

No $2^{\circ}$ trimestre de 2016, após o rompimento da barragem, a água ainda estava contaminada por substâncias tóxicas e, no decorrer do ano, a maioria das amostras de água se enquadrou na classificação baixa. Essa baixa contaminação pode favorecer a proliferação da biota do fitoplâncton, fazendo com que o Índice de Estado Trófico na maioria das estações subisse durante o período avaliado e atingisse a classificação mesotrófica $(52<$ IET $\leq 59)$, inferior quando comparado aos trimestres anteriores. Para o IET, a Análise de Variância também apresentou diferenças significativas $(\mathrm{F}=7,32 ; \mathrm{p}<0,0001)$; contudo o teste de Tukey indicou que estas ocorrem entre $4^{\circ}$ trimestre de $2014,3^{\circ}$ e $4^{\circ}$ trimestres de 2016 e $1^{\circ}$ trimestre de 2017, em relação aos demais.

Em relação ao IQA, foi observado um aumento nos valores obtidos, se aproximando daqueles de antes do desastre (Tabela 2). Quando foi comparada a média dos valores de IQA obtidos das análises de água das 13 estações de monitoramento entre o $3^{\circ}$ e o $4^{\circ}$ trimestre, percebeu-se uma diminuição de $8,19 \%$, o que também ocorreu no ano anterior quando foi observada uma queda média de $15,00 \%$ no mesmo período anual. Tal fator, além de ser 
explicado pela influência do desastre da Samarco em 2015, teve como auxílio também a variação de temperatura, assim como em 2016, visto que no último trimestre do ano há um aumento com a chegada do verão. A variação da temperatura é um dos fatores para o cálculo do IQA, possuindo o quarto maior peso para o cálculo.

\subsection{Relação entre perda da qualidade da água e a epidemia de febre amarela na Bacia do Rio Doce}

A Análise de Componentes Principais (ACP) entre os índices de qualidade de água monitorados pelo IGAM e os dados epidemiológicos da SES-MG é apresentada na Tabela 4 e Figura 3. A porcentagem de explicação da variância nos eixos 1, 2 e 3 foi de 40,46, 31,27 e $18,78 \%$, respectivamente. Apesar de apresentar boa explicação acumulada nos três eixos $(90,7 \%)$ não foi possível verificar uma clara relação entre os parâmetros de qualidade de água (IQA, IET, CT) e os dados epidemiológicos (CN e CC).

Tabela 4: Correlação das variáveis com os eixos da Análise de Componentes Principais entre os parâmetros de qualidade de água e os dados epidemiológicos de febre amarela da bacia do Rio

Doce (valores em negrito representam correlações significativas com os fatores $(\mathrm{p}<0,05)$ )

\begin{tabular}{cccc}
\hline & Fator 1 & Fator 2 & Fator 3 \\
\hline IQA & $-0,089322$ & $-\mathbf{0 , 8 5 7 4 6 3}$ & 0,180559 \\
IET & $-0,264126$ & $-0,247945$ & $-0,931577$ \\
CT & 0,174911 & $\mathbf{0 , 8 5 0 0 2 8}$ & $-0,126652$ \\
CN & $-\mathbf{0 , 9 7 9 2 3 4}$ & 0,150918 & 0,095778 \\
CC & $-\mathbf{0 , 9 7 7 7 6 2}$ & 0,146227 & 0,116577 \\
\hline
\end{tabular}

IQA = Índice de Qualidade de Água; IET = Índice de Estado Trófico; $\mathrm{CT}=$ Contaminação por Tóxicos; $\mathrm{CN}=$ Casos notificados; $\mathrm{CC}=$ Casos confirmados de febre amarela

Fonte: Autoria própria

A correlação do IQA é negativa com o eixo 2, pois este índice varia de 0 a 100, sendo os valores até 25 considerados de qualidade Muito Ruim e aqueles acima de 90, Excelente. Opostamente, o CT apresentou correlação positiva com este eixo, pois valores baixos $(\mathrm{CT}<$ 1,2 concentração limite) representam boa qualidade de água, enquanto valores altos (CT > 2,0 concentração limite) representam má qualidade. 
Figura 3: Análise de Componentes Principais entre os parâmetros de qualidade de água e os dados epidemiológicos de febre amarela da bacia do Rio Doce

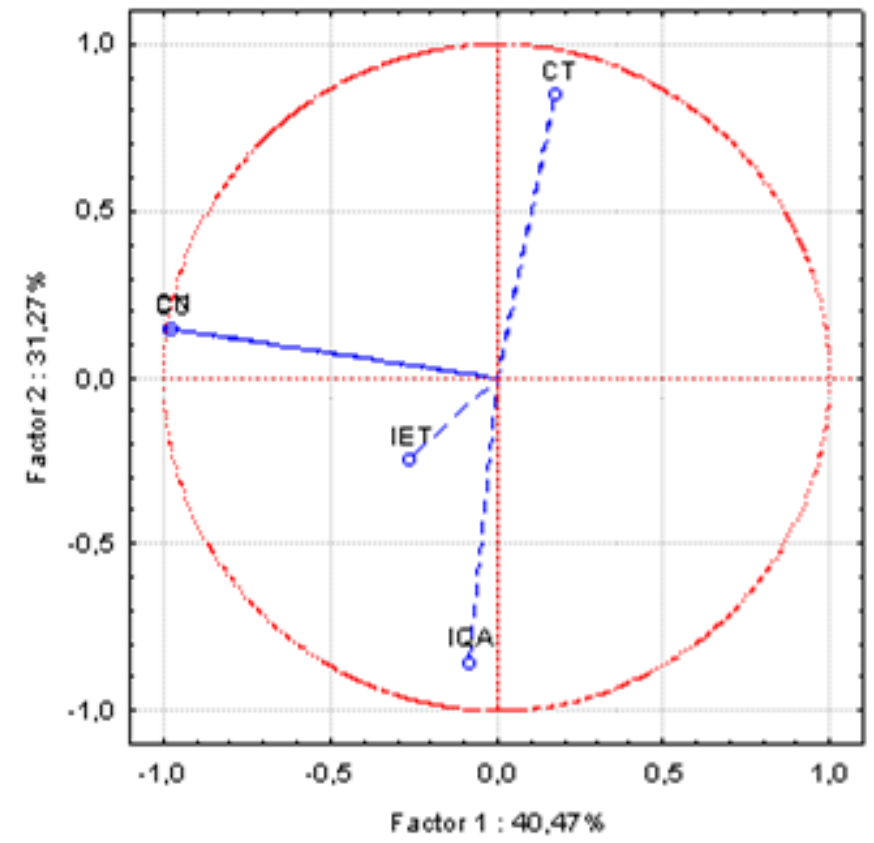

IQA = Índice de Qualidade de Água; IET = Índice de Estado Trófico;

$\mathrm{CT}=$ Contaminação por Tóxicos; $\mathrm{CN}=$ Casos notificados; $\mathrm{CC}=$ Casos confirmados de febre amarela

Fonte: Autoria própria

É possível constatar que a qualidade da água foi significativamente correlacionada aos eixos 1 e 3; enquanto os casos de febre amarela estavam correlacionados ao eixo 2. Uma vez que os eixos 1, 2 e 3 são perpendiculares entre si, o não alinhamento das variáveis reflete a ausência de uma relação direta entre esses parâmetros.

Ao verificar por matriz de correlação os pontos específicos com maiores números de casos de febre amarela (RD023, RD035, RD045, RD053, RD058, RD059, RD067), apenas para o IET e os casos notificados foi observada correlação positiva e significativa $(r=0,881$; p < 0,01) em RD035, na região de Coronel Fabriciano. Ou seja, um maior número de casos notificados ocorreu com a piora da qualidade, quando este índice apontou águas hipereutróficas.

Analisando dados do Sistema de Informações do Programa Nacional de Imunizações (SIPNI), contidos na Tabela 5, a média da cobertura vacinal do estado de Minas Gerais até dezembro de 2016 era de 57,26\%, muito abaixo da média preconizada. Durante a epidemia, com as campanhas de vacinação realizadas, essa média chegou a 80,64\%. 
Tabela 5: Cobertura Vacinal Acumulada - Minas Gerais, 2016 e 2017

\begin{tabular}{|c|c|c|}
\hline Regional & $\begin{array}{c}\text { Cobertura vacinal } \\
\text { acumulada } 2007-2016(\%)\end{array}$ & $\begin{array}{c}\text { Cobertura vacinal } \\
\text { acumulada } 2007 \text { - } 2017(\%)\end{array}$ \\
\hline Alfenas & 58,97 & 74,04 \\
\hline Barbacena & 55,53 & 77,27 \\
\hline Belo Horizonte & 54,87 & 79,92 \\
\hline Coronel Fabriciano & 51,34 & 80,28 \\
\hline Diamantina & 58,51 & 78,36 \\
\hline Divinópolis & 64,22 & 84,36 \\
\hline Governador Valadares & 54,09 & 84,99 \\
\hline Itabira & 66,87 & 94,48 \\
\hline Ituiutaba & 59,52 & 71,64 \\
\hline Januária & 70,74 & 94,28 \\
\hline Juiz de Fora & 46,37 & 82,68 \\
\hline Leopoldina & 47,14 & 71,38 \\
\hline Manhumirim & 43,67 & 84,06 \\
\hline Montes Claros & 56,77 & 81,57 \\
\hline Passos & 56,92 & 72,80 \\
\hline Patos de Minas & 73,29 & 83,76 \\
\hline Pedra Azul & 46,74 & 72,40 \\
\hline Pirapora & 66,71 & 89,96 \\
\hline Ponte Nova & 46,77 & 69,25 \\
\hline Pouso Alegre & 49,75 & 65,23 \\
\hline São João Del Rey & 48,80 & 66,74 \\
\hline Sete Lagoas & 61,40 & 80,33 \\
\hline Teófilo Otoni & 52,70 & 98,04 \\
\hline Ubá & 56,88 & 76,09 \\
\hline Uberaba & 67,85 & 86,42 \\
\hline Uberlândia & 72,57 & 85,76 \\
\hline Unaí & 88,94 & 101,99 \\
\hline Varginha & 51,00 & 73,62 \\
\hline Minas Gerais & 57,26 & 80,64 \\
\hline
\end{tabular}

Fonte: http://pni.datasus.gov.br (2017).

\section{DISCUSSÃO}

Considerando os dados epidemiológicos da febre amarela coletados nesse estudo, foi observado que os municípios com epizootias permaneceram silenciosos no início da epidemia devido ao tempo hábil necessário para a correta investigação da causa mortis do animal. Com o passar dos meses, o número de casos confirmados foi aumentando devido ao intenso monitoramento das autoridades em saúde.

No âmbito da saúde pública, sabe-se que há um modelo de descentralização administrativa, que busca repartir atribuições a cada ente, de maneira a tornar mais eficiente e ágil a gestão das ações (MINISTÉRIO DA SAÚDE, 2017). Um exemplo desse modelo são 
as 28 Superintendências ou Gerências Regionais de Saúde existentes no estado de Minas Gerais. Vale salientar que destas 28 Gerências Regionais, 25 apresentaram casos suspeitos ou confirmados de febre amarela no ano de 2017.

Inicialmente pretendia-se traçar o fluxo de avanço da doença a partir do primeiro município infectado, para então ser possível chegar a respostas sobre a relação do desastre ambiental causado pelo rompimento da barragem em Mariana com o surto epidemiológico observado em Minas Gerais. Porém, devido à falta de detalhamento de alguns dados fornecidos pelos órgãos oficiais, não foi possível traçar esse fluxo. Ressalta-se também a grande dificuldade em se obter dados precisos, devido à demora na atualização de algumas plataformas governamentais.

Houve um aumento da preocupação das autoridades em saúde devido à possibilidade da reintrodução da febre amarela urbana na região Sudeste do Brasil, uma vez que surtos epidêmicos foram observados em centros urbanos com mais de 100 mil habitantes, como em Teófilo Otoni, Coronel Fabriciano e Governador Valadares. Nesses municípios há outros vetores, como o A. aegypti, a pressão do desmatamento e da caça sobre os remanescentes de Mata Atlântica, além da influência de fatores ambientais sazonais que propiciam o aumento da densidade vetorial. Fatores estes que contribuem para o aumento de casos da febre amarela nessas regiões (CAVALCANTE \& TAUIL, 2017).

A degradação dos ecossistemas aquáticos e das interações entre as espécies que vivem nesses ambientes pode ter intensificado o aumento do número de casos da febre amarela. Além do dano físico causado pela lama liberada, também houve adição de substâncias tóxicas, que quando em contato com a biota, alteram o crescimento, a taxa de reprodução e a sobrevivência das espécies, impedindo a proliferação de organismos devido ao aumento de toxicidade (LOPES, 2016). Este efeito é evidenciado no presente estudo, uma vez que foi observada redução nos valores de IQA e alta contaminação por tóxicos após o rompimento da barragem de Fundão.

Apesar da elevada turbidez provocada pelo lançamento da lama no curso d'água, o IET não considera somente a transparência da água, mas também concentrações de clorofilaa e fósforo total (LAMPARELLI, 2004). A atividade fotossintética foi afetada pela menor entrada de luz no ambiente aquático, bem como o carreamento e/ou soterramento das algas, reduzindo as concentrações de clorofila-a. Por outro lado, a atividade mineradora provoca a liberação de nutrientes (especialmente fósforo associado ao ferro) para a coluna d'água, o que aumenta sua concentração e, consequentemente, eleva a produtividade primária local, 
causando eutrofização do ambiente aquático (GOMES et al., 2000). Dessa forma, diferentes valores dessas variáveis compensaram o IET, havendo pouca mudança neste índice.

Uma das hipóteses para a investigação da relação do surto epidemiológico da febre amarela com o desastre de Mariana seria verificar se houve aumento da população do vetor Haemagogus spp. na região da bacia hidrográfica do Rio Doce, uma vez que alterações físico-químicas foram observadas no corpo hídrico após o desastre. Foi observado no presente estudo que a ACP não demonstrou correlação dos índices de qualidade de água com os dados epidemiológicos, sendo assim umas das explicações para este resultado é o fato das larvas do Haemagogus spp. se desenvolverem em ocos de árvores e reservatórios de bromélias, onde comumente há ausência de predadores (MÜLLER et al., 2010; SILVA; LOZOVEI, 1999).

Portanto, a associação direta entre a qualidade do corpo hídrico e o aumento da população do vetor não existe, visto que o impacto causado pelo rompimento da barragem não atingiu diretamente os ocos de árvores que são os pontos de oviposição do mosquito. Buscando então entender o porquê do aumento dos casos de febre amarela após esse desastre ambiental esse estudo analisou também quais seriam os possíveis predadores das larvas do mosquito vetor da febre amarela.

Foi observado na literatura que macroinvertebrados bentônicos são predadores tanto das formas larvais quanto de adultos de mosquitos hematófagos. A bacia do Rio Doce é uma das mais diversas em relação ao número de táxons (93 táxons), sendo comparável à do Rio São Francisco, que possui 83 táxons nos seus respectivos trechos dentro do estado de Minas Gerais (FRANÇA \& CALLISTO, 2007; MAIA-BARBOSA et al., 2009). Essa fauna inclui espécimes de diversos grupos, como anelídeos, platelmintos, copépodes, anfíbios, répteis, peixes, aves e insetos (especialmente as ordens Hemiptera, Coleoptera, Diptera e Odonata) (ANDRADE \& SANTOS, 2004).

Os indivíduos do grupo Odonata, representado pelas libélulas, são encontrados em todos os tipos de ambientes aquáticos de água doce e também em águas salobras, tanto na zona temperada como na tropical. Entretanto, algumas espécies são restritas a ambientes tropicais, como as fitotelmas ou fitotelmata, que são pequenos corpos d'água contidos em cavidades de plantas como ocos de árvores, bambus e bromélias (PERUQUETTI, 2004). As ninfas de libélulas têm sido registradas como predadoras mais eficientes de larvas de mosquitos que habitam esses recintos, principalmente quando em seu último estágio, podendo predar cerca de 6 larvas/dia e, quando encontram populações densas da presa, 
matam mais do que conseguem comer (ANDRADE \& SANTOS, 2004). Já os adultos de libélulas são predadores de mosquitos e moscas (GOULART \& CALLISTO, 2003), sendo importantes no controle desses vetores de doenças.

Nesse sentido, pode-se inferir que a lama liberada durante o rompimento da barragem, além de causar alterações físico-químicas na água, pode ter causado também uma diminuição nas populações de larvas e adultos de libélulas e outros insetos bentônicos que habitam a calha central do Rio Doce.

Dessa forma, é possível que o desastre ambiental tenha causado um desequilíbrio na cadeia alimentar da bacia, propiciando uma diminuição na quantidade dos predadores de larvas do mosquito vetor da febre amarela como, por exemplo, libélulas e insetos que se desenvolvem no ambiente bentônico. Isso acarretaria um aumento crescente no número das larvas do vetor da febre amarela e, consequentemente, aumento da doença na região.

Gomes et al. (2010) explica que a acrodendrofilia, tendência de certos insetos silvestres em viver no topo ou na copa de árvores, representa uma parte do caráter do comportamento do Haemagogus spp., uma vez que já foi observado sua presença ao nível do solo. Essa aproximação se justifica pelo fato de que riachos e córregos podem ser utilizados como criadouros naturais (FERNANDES, 2011), assim como porções hídricas mais calmas. Neste ambiente, o controle biológico dos mosquitos está relacionado aos peixes larvófagos. Muitos estudos demonstram que, além do consumo efetivo das larvas dos vetores logo após a eclosão, esses peixes possuem capacidade de repelir a oviposição das fêmeas de mosquitos, liberando substâncias na água (PAIVA, 2013; PEREIRA; OLIVEIRA, 2014). Assim, a obstrução das brânquias dos peixes pelo excesso de turbidez da água, dentre outros motivos provenientes da presença da lama no corpo hídrico, diminuiu a ocorrências desses larvófagos (CHAVES et al., 2016), o que favoreceria a permanência e aumento do vetor, contribuindo assim para o consequente aumento no número de casos da doença na região.

Assim como os Aedes, os Haemagogus não depositam seus ovos diretamente na superfície aquática, mas sim na parede interna do criadouro próximo à lâmina d'água. Quando os ovos são submersos, as larvas eclodem e passam a se desenvolver, se alimentando da matéria orgânica presente na água, até se tornarem pupas. Cerca de sete a dez dias após a eclosão dos ovos, os Haemagogus chegam à fase de mosquitos adultos. Já os Sabethes lançam seus ovos diretamente sobre a superfície da água e chegam à fase adulta quase um mês depois (FUNDAÇÃO OSWALDO CRUZ, 2017a). Por esse mecanismo, a maior resistência dos ovos de Haemagogus nos períodos de seca aumenta suas chances de 
sobrevivência. Consequentemente, mais indivíduos chegam à idade adulta quando as condições se tornam propícias novamente, aumentando as probabilidades de surtos epidêmicos durante o verão, uma vez que nesse período ocorre um aumento da reprodução desses vetores.

As pesquisas realizadas na bacia do Rio Doce após o rompimento da barragem de Fundão ainda não apresentaram dados publicados acerca do impacto sobre a comunidade de macroinvertebrados bentônicos e demais organismos aquáticos (IGAM, 2017). Entretanto, o presente estudo defende a hipótese de que esse desastre ocasionou grande diminuição da fauna de macroinvertebrados bentônicos e vertebrados aquáticos (peixes e anfíbios), indivíduos estes que possuem grande potencial para controle biológico de larvas e adultos de mosquitos hematófagos, vetores de doenças como a febre amarela. A causa imediata dessa dizimação foi o soterramento dos organismos pelo rejeito de maior densidade, enquanto aqueles que sobreviveram a isso, possivelmente, morreram devido ao colabamento das brânquias, que os levou à morte por asfixia por meio dos sólidos finos em suspensão (IBAMA, 2015).

Associado com a baixa qualidade da água apresentada neste estudo, desde 2012 diferentes municípios do Sudeste brasileiro têm se deparado com reduções da pluviosidade, delineando um cenário complexo de escassez hídrica. Esse fenômeno climático contribui para o agravamento da oferta de água em bacias hidrográficas, em razão da baixa disponibilidade hídrica qualitativa e/ou quantitativa (ANA, 2014). A região próxima ao Rio Doce já apresentava danos ambientais causados pela mineração, como a destruição das áreas preservadas, fazendo com que os animais encontrassem dificuldades de adaptação, sobretudo quanto à alimentação (FUNDAÇÃO OSWALDO CRUZ, 2017c; SURTO, 2017).

Dessa forma, a menor pluviosidade e os impactos provocados pelo rejeito na calha central e nas matas ciliares do Rio Doce causaram uma redução considerável da oferta de alimento nos remanescentes de Mata Atlântica, de modo que primatas desnutridos e possivelmente imunodeprimidos estivessem mais vulneráveis à doença. Já no final de 2016, mortes de primatas associadas à essa epizootia, especialmente muriquis e bugios, foram registradas em pelo menos duas reservas na região (RPPNs Feliciano Miguel Abdala e Mata do Sossego) (STRIER et al., 2018).

Além dessa vulnerabilidade, a proximidade cada vez maior das áreas urbanas em relação às áreas verdes remanescentes propicia um aumento da área onde comumente ocorre a transmissão da doença. A Floresta Atlântica, onde está localizada $98 \%$ da bacia 
hidrográfica do Rio Doce, já perdeu mais de 93\% de sua área, restando menos de 100.000 $\mathrm{km}^{2}$ de vegetação remanescente, provenientes do processo de ocupação do território brasileiro para exploração de recursos naturais, o qual ocasiona a supressão da vegetação nativa (DE FREITAS PEREIRA, 2016; TABARELLI et al., 2005). Este crescente avanço do processo de ocupação em direção às áreas antes vegetadas pode fazer com que animais já doentes se aproximem dos centros urbanos, possibilitando que vetores antes não infectados adquiram o vírus.

Outro fator que pode ter contribuído para o aumento crescente no número da febre amarela é o modo de vida do mosquito Haemagogus spp que apresenta atividade diurna, sendo também encontrado nas bordas de fragmentos florestais, o que favorece a transmissão da febre amarela aos humanos que acessam estas matas (VALE et al., 2018). Considerando os dados supracitados, somados ao fato de que predadores de mosquitos tiveram suas populações drasticamente reduzidas pelo rejeito de minério no Rio Doce, o aumento nas populações de Haemagogus spp pode ter causado o seu deslocamento para vegetações periurbanas em busca de alimento, contribuindo também para o aumento da doença.

Outro fator relevante em relação à febre amarela, diz respeito à taxa de imunização dos habitantes do estado de Minas Gerais no início do ano de 2017. A prevenção dessa doença se dá através de vacinação, cujas doses são oferecidas gratuitamente por meio do Sistema Único de Saúde (SUS). Segundo informações da Secretaria de Estado de Saúde (2017), a partir do início de 2017, cada indivíduo deve receber pelo menos uma dose da vacina ao longo da vida para ser considerado imunizado. Porém, para que a vacinação consiga prevenir a ocorrência de surtos, é necessário que a cobertura vacinal da população tenha uma taxa de no mínimo 95\%. Porém, os dados apresentados demonstraram que, até o ano de 2016, todas as regionais de saúde do Estado de Minas Gerais se encontravam com cobertura vacinal abaixo da taxa preconizada pelo Ministério da Saúde, o que pode ter contribuído para que a população estivesse mais suscetível ao vírus. Durante a epidemia da febre amarela em 2017, houve grande mobilização para a vacinação da população, mas mesmo assim, foi verificado que os índices ainda se encontravam abaixo do valor preconizado. Destaca-se que apesar de ser o estado que mais recebeu doses da vacina durante a epidemia, a distribuição não foi homogênea no território de Minas Gerais. Foi observado ainda que foram baixos os valores per capita investidos em vigilância epidemiológica (FGV, 2017), o que interfere também no aumento de casos da doença. 
Os danos ocasionados por um desastre ambiental da magnitude do acidente da Samarco continuarão a causar impactos nos locais afetados por muitos anos, danos estes que, por vezes, só se tornarão evidentes com o passar do tempo. Por este motivo, acredita-se que o presente estudo, assim como outros que venham a ser realizados na região, poderão fornecer dados que futuramente serão fundamentais para novas conclusões a respeito da associação do rompimento da barragem com o aumento dos casos de febre amarela e outras enfermidades que venham a ser observadas após o desastre. Em função disso, é fundamental manter o monitoramento ambiental e epidêmico na região, além de se realizar ações preventivas de conscientização popular em relação à febre amarela e demais doenças endêmicas.

\section{CONCLUSÕES}

No âmbito epidemiológico, ao analisar o aumento de uma doença, é necessário considerar em conjunto fatores ambientais, sociais e antrópicos. Dessa forma, o crescente número de casos de febre amarela na Bacia do Rio Doce deve ser avaliado considerando-se os diversos aspectos que, em conjunto, podem ter propiciado o reaparecimento dessa doença na região.

O presente estudo não detectou correlação direta entre os índices de qualidade de água e o aumento dos casos da febre amarela. Porém, a análise desses índices em conjunto com a avaliação de outros fatores ambientais e epidemiológicos discutidos neste trabalho sugere uma ação indireta do dano ambiental causado sobre a ocorrência da epidemia de febre amarela.

Salienta-se que novos trabalhos relacionados à região de estudo serão fundamentais para a determinação do nível de participação do dano ambiental em relação à epidemia ocorrida.

\section{REFERÊNCIAS}

ANA. Agência Nacional de Águas. Conjuntura dos recursos hídricos no Brasil - Encarte Especial sobre a Crise Hídrica. 2014. Disponível em. Acesso em: 23 nov 2017.

ANDRADE, Carlos Fernando S.; SANTOS, Luciana Urbano dos. O uso de predadores no controle biológico de mosquitos, com destaque aos Aedes. Instituto de Biologia. Universidade Estadual de Campinas - UNICAMP - SP, 2004. 
CAMARGO-NEVES, Vera. L. F. de; POLETTO, Daniela W.; RODAS, Lílian A. C.; PACHIOLI, Márcio L.; CARDOSO, Rubens P.; SCANDAR Sirle A. S.; SAMPAIO, SusyM. P.; KOYANAGUI, Paulo H.; BOTTI, Mauricio V.; MUCCI, Luis F.; GOMES, Almério de C. Entomological investigation of a sylvatic yellow fever area in São Paulo State, Brazil. Cad. Saúde Pública, Rio de Janeiro, v. 21, n. 4, p. 1278-1286, ago. 2005.

CAVALCANTE, Karina Ribeiro Leite Jardim; TAUIL, Pedro Luiz. Risco de reintrodução da febre amarela urbana no Brasil. Epidemiol. Serv. Saude, Brasília, v. 26, n. 3, p. 617 620, jul-set, 2017.

CHAVES, Bárbara Regina Neves; AMARANTE, Marcelo Coutinho; NACIF, Wanderlene Ferreira. Mortandade de peixes na Bacia do Rio Doce após rompimento da barragem da Samarco no distrito de Bento Rodrigues (Mariana/MG), em 05/11/2015. Belo Horizonte, Sistema Estadual de Meio Ambiente e Recurso Hídrico, 2016.

COSTA, Isabelle Matos Pinheiro; CALADO, Daniela Cristina. Incidência dos casos de dengue (2007-2013) e distribuição sazonal de culicídeos (2012-2013) em Barreiras, Bahia. Epidemiol. Serv. Saúde, Brasília, v. 25, n. 4, p. 735-744, dez. 2016.

COSTA, Raissa de Moura; FACIOLI; Larissa de Souza; REIS, Thainá Desiree Franco dos; SÁ, Odila Rigolin de; BERNARDES, Nicole Blanco. Febre Amarela: Sua Perspectiva No Brasil. Id on Line Rev.Mult. Psic., vol.12, n.41, p.435-448, jun. 2018.

DATASUS. Si-PNI - Sistema de Informações do Programa Nacional de Imunizações. Disponível em: http://pni.datasus.gov.br. Acesso em: 22 nov. 2017.

DE FREITAS PEREIRA, Bruno Wendell; MACIEL, Maria de Nazaré Martins; OLIVEIRA, Francisco de Assis; ALVES, Marcelo Augusto Moreno da Silva; RIBEIRO, Adriana Melo; FERREIRA, Bruno Monteiro; RIBEIRO, Ellen Gabriele Pinto. Uso da terra e degradação na qualidade da água na bacia hidrográfica do rio Peixe-Boi, PA, Brasil/Land use and water quality degradation in the Peixe-Boi River watershed. Revista Ambiente \& Água, v. 11, n. 2, p. 472, 2016.

FERNANDES, Gláucia de Oliveira. Culicídeos vetores em uma unidade de conservação da Caatinga na região do Seridó no Rio Grande do Norte - aspectos da transmissão de doenças. 2011. 121 f. Dissertação (Mestrado em Desenvolvimento e Meio Ambiente) Universidade Federal do Rio Grande do Norte. Centro de Biociências, Natal, 2011.

FGV - Fundação getúlio Vargas. 2017. Febre amarela no Brasil: um estudo de caso. Rio de Janeiro: FGV. 39p.

FRANÇA, Juliana Silva; CALLISTO, Marcos. Coleção de macroinvertebrados bentônicos: ferramenta para o conhecimento da biodiversidade em ecossistemas aquáticos continentais. Neotropical Biology and Conservation, v. 2, n. 1, p. 3-10, 2007.

FUNDAÇÃO OSWALDO CRUZ. Conheça semelhanças e diferenças entre mosquitos transmissores da febre amarela. Rio de Janeiro, 2017. Disponível em: https://portal.fiocruz.br/noticia/conheca-semelhancas-e-diferencas-entre-mosquitostransmissores-da-febre-amarela. Acesso em: 28 jul. 2020. 
FUNDAÇÃO OSWALDO CRUZ. Febre amarela: sintomas, transmissão e prevenção. Rio de Janeiro, 2017. Disponível em: https://www.bio.fiocruz.br/index.php/febre-amarelasintomas-transmissao-e-prevencao. Acesso em: 10 abr. 2017.

FUNDAÇÃO OSWALDO CRUZ. Surto de febre amarela no Brasil é destaque na Revista Radis. Rio de Janeiro. 2017. Disponível em: https://portal.fiocruz.br/ptbr/content/surto-de-febre-amarela-no-brasil-e-destaque-na-radis. Acesso em: 10 abr. 2017.

GOMES, Almério de Castro; TORRES, Maria Amélia Nascimento; Paula, Marcia Bicudo de; FERNANDES, Aristides; MARASSÁ, Ana Maria; CONSALES, Cleide Aschenbrenner; FONSECA, Daltro Fernandes. Ecologia de Haemagogus e Sabethes (Diptera: Culicidae) em áreas epizoóticas do vírus da febre amarela, Rio Grande do Sul, Brasil. Epidemiol. Serv. Saúde, Brasília, v. 19, n. 2, p. 101-113, jun. 2010.

GOULART, Michael Dave C.; CALLISTO, Marcos. 2003. Bioindicadores de qualidade de água como ferramenta em estudos de impacto ambiental. Revista da FAPAM, v. 1, p. 1-9.

IBAMA - Instituto Brasileiro do Meio Ambiente e dos Recursos Naturais Renováveis. 2015. Laudo Técnico Preliminar - Impactos ambientais decorrentes do desastre envolvendo o rompimento da barragem de Fundão, em Mariana, Minas Gerais. Diretoria de Proteção Ambiental - DIPRO. Coordenação Geral de Emergências Ambientais - CGEMA. Brasília: MMA, 74p.

IGAM - Instituto Mineiro de Gestão das Águas. 2015a. Monitoramento da Qualidade das Águas Superficiais do Rio Doce no Estado de Minas Gerais. Relatório Trimestral $-3^{\circ}$ trimestre de 2015. Belo Horizonte: SEMAD.

IGAM - Instituto Mineiro de Gestão das Águas. 2015b. Monitoramento da Qualidade das Águas Superficiais do Rio Doce no Estado de Minas Gerais. Relatório Trimestral $-4^{\circ}$ trimestre de 2015. Belo Horizonte: SEMAD.

IGAM - Instituto Mineiro de Gestão das Águas. 2016a. Monitoramento da Qualidade das Águas Superficiais do Rio Doce no Estado de Minas Gerais. Relatório Trimestral $-1^{\circ}$ trimestre de 2016. Belo Horizonte: SEMAD.

IGAM - Instituto Mineiro de Gestão das Águas. 2016b. Monitoramento da Qualidade das Águas Superficiais do Rio Doce no Estado de Minas Gerais. Relatório Trimestral $-2^{\circ}$ trimestre de 2016. Belo Horizonte: SEMAD.

IGAM - Instituto Mineiro de Gestão das Águas. 2016c. Monitoramento da Qualidade das Águas Superficiais do Rio Doce no Estado de Minas Gerais. Relatório Trimestral $-3^{\circ}$ trimestre de 2016. Belo Horizonte: SEMAD.

IGAM - Instituto Mineiro de Gestão das Águas. 2016d. Monitoramento da Qualidade das Águas Superficiais do Rio Doce no Estado de Minas Gerais. Relatório Trimestral $-4^{\circ}$ trimestre de 2016. Belo Horizonte: SEMAD.

IGAM - Instituto Mineiro de Gestão das Águas. 2017. Monitoramento da Qualidade das Águas Superficiais do Rio Doce no Estado de Minas Gerais. Relatório Técnico - 
Acompanhamento da Qualidade das Águas do Rio Doce Após o Rompimento da Barragem da Samarco no distrito de Bento Rodrigues - Mariana/MG. Belo Horizonte: SEMAD.

LAMPARELLI, M. C. Graus de trofia em corpos d'água do Estado de São Paulo. 2004. Tese de Doutorado. Ph. D. Thesis, University of São Paulo, State of São Paulo, Brazil, 3 de Setembro.

LOPES, Luciano Motta Nunes. O rompimento da barragem de Mariana e seus impactos socioambientais. Sinapse Múltipla, belo Horizonte, v. 5, n. 1, p. 1-14, jun , 2016.

MAIA-BARBOSA, P. M.; MACHADO, C. F.; BARBOSA, F. A. R.; FERREIRA, H. L. M.; BRITO, S. L.; JUNQUEIRA, M. V.; CAMPOS, M. S.; MEYER, S. T.; JARDIM, B. F. M.; GAMA, F. O.; MOTA, H. R.; MENEDEZ, R. M. 2009. Diversidade de Organismos Aquáticos In: BIOTA MINAS - Diagnóstico do Conhecimento sobre a Biodiversidade no estado de Minas Gerais - Subsídio ao Programa BIOTA MINAS. Belo Horizonte: Fundação Biodiversitas, p.81-118.

MINISTÉRIO DA SAÚDE, 2017. Febre Amarela. Disponível em: http://bvsms.saude.gov.br/bvs/febreamarela/historico.php. Acesso em: 22 mai. 2017.

MINISTÉRIO DA SAÚDE, 2017. Febre Amarela. Disponível em: http://portalsaude.saude.gov.br/index.php/o-ministerio/principal/secretarias/svs/febreamarela. Acesso em: 11 mai. 2017.

MINISTÉRIO DA SAÚDE, 2017. Secretaria de Vigilância em Saúde. Centro de Operações de Emergências em Saúde Pública - Informe no 40/2017. Disponível em: http://portalarquivos.saude.gov.br/images/pdf/2017/maio/12/COES-FEBREAMARELA--INFORME-40---Atualiza----o-em-11maio2017.pdf. Acesso em: 16 mai. 2017.

MINISTÉRIO DA SAÚDE, 2017. Situação Epidemiológica / Dados. Disponível em: http://portalsaude.saude.gov.br/index.php/situacao-epidemiologica-dados-febreamarela.

Acesso em: 02 mai. 2017.

MÜLLER, Gerson Azulim; MARCONDES, Carlos Brisola; NAVARRO-SILVA, Mário Antônio. Aplicação de marcadores microssatélites para o estudo de Culicidae (Diptera): revisão com especial referência a Haemagogus. Boletín de Malariología y Salude Ambiental, v. 2, p. 175-186, 2010.

MYERS, Norman; MITTERMEIER, Russell A.; MITTERMEIER, Cristina G., et al., 2000. Biodiversity hotspots for conservation priorities. Nature 403, 853-858.

PAIVA, Clemison Nogueira. Utilização de peixes larvófagos para o controle das formas imaturas do $\boldsymbol{A}$. aegypti em depósitos domiciliares de água. 2013. 99 f. Dissertação (Mestrado em Saúde Pública) - Universidade Federal do Ceará. Faculdade de Medicina, Fortaleza, 2013.

PEREIRA, Boscolli Barbosa; OLIVEIRA, Eidis Antônio de. Determinação do potencial larvófago de Poecilia reticulata em condições domésticas de controle biológico. Cad. saúde colet.. Rio de Janeiro, v. 22, n. 3, p. 241-245, Set. 2014 
NEVES, David Pereira; MELO, Alan Lane de; LINARDI, Pedro Marcos; VITOR, Ricardo W.Almeida. Parasitologia humana. 11 a ed. São Paulo: Editora Atheneu. 2005. p 370.

PERUQUETTI, Patrícia Santos Ferreira et al. Odonata (libélulas) do município de Luís Antônio, São Paulo, Brasil: relação com o uso do solo e riqueza faunística. 2004. 62 f. Tese (Doutorado em Ciências Biológicas) - Universidade Federal de São Carlos, São Carlos, 2004).

REY, Luis. Parasitologia. $3^{a}$ ed. Rio de Janeiro: Guanabara Koogan, 2001.

SANTOS, Vanessa Sardinha dos. Impactos ambientais do acidente em Mariana (MG). Brasil Escola. Disponível em: http://brasilescola.uol.com.br/biologia/impactos-ambientaisacidente-mariana-mg.htm. Acesso em: 09 abr. 2017.

SEDRU - Secretaria de Estado de Desenvolvimento Regional, Política Urbana e Gestão Metropolitana. 2016. Avaliação dos efeitos e desdobramentos do rompimento da Barragem de Fundão em Mariana - MG. Belo Horizonte, 03 de fevereiro de 2016. Disponível http://www.agenciaminas.mg.gov.br/ckeditor_assets/attachments/770/relatorio_final_ft_03 _02_2016_15h5min.pdf. Acesso em: 01 mai. 2017.

SES - Secretaria de Estado de Saúde. Atualização sobre a investigação de casos suspeitos de febre amarela silvestre. Minas Gerais, 2017. Disponível em: http://www.saude.mg.gov.br/component/search/?all=febre+amarela+informe+epidemiol\% C3\%B3gico\&area=all. Acesso em: 22 mai. 2017.

SILVA, Mario Antônio Navarro da; LOZOVEI, Ana Leuch. Ocorrência de Haemagogus (Conopostegus) leucocelaenus (Dyar \& Shannon) e Toxorhynchites (Lynchiella) theobaldi (Dyar \& Knab) em ocos de árvore em capão de mata, Curitiba, Paraná, Brasil. Rev. Bras. Zool., Curitiba, v. 16, supl. 1, p. 257-267, 1999.

STRIER, Karen B; TABACOW, Fernanda P; POSSAMAI, Carla B; FERREIRA, Anderson I G; NERY, Marcello S; MELO, Fabiano R; MENDES, Sérgio L. Status of the northern muriqui (Brachyteles hypoxanthus) in the time of yellow fever. Primates; v. 60, n. 1, p. 21-28, 2018.

TABARELLI, Marcelo; PINTO, Luiz Paulo; SILVA, José Maria C.; HIROTA, Márcia M.; BEDÊ, Lúcio C. Desafios e oportunidades para a conservação da biodiversidade na Mata Atlântica brasileira. Megadiversidade, v. 1, n. 1, p. 132-138, 2005.

VALE, Caroline Almeida; JUNIOR, Vicente Sanches; PREZOTO, Fábio. Febre Amarela: o Mosquito, o Macaco e o Ser Humano. CES Revista, v. 32, n. 2, p. 7-27, 2018.

VASCONCELOS, Pedro Fernando da Costa. Febre Amarela. Revista da Sociedade Brasileira de Medicina Tropical, v. 36, n. 2, p. 275-293, 2003. 